\title{
An activities-based course in optics for non-technical majors
}

Jack Glassman, Rebecca Lindell

Jack Glassman, Rebecca Lindell, "An activities-based course in optics for non-technical majors," Proc. SPIE 9665, Tenth International Topical Meeting on Education and Training in Optics and Photonics, 96651I (3 June 2007); doi: $10.1117 / 12.2207325$

SDIE Event: Tenth International Topical Meeting on Education and Training in Optics and Photonics, 2007, Ottawa, Ontario, Canada 


\title{
An Activities-Based Course in Optics for Non-technical Majors
}

\author{
Jack Glassman and Rebecca Lindell \\ Department of Physics, Southern Illinois University Edwardsville, Edwardsville, Illinois 62026-1654, USA \\ Phone: 618-650-2035, e-mail: jacglas@siue.edu
}

\begin{abstract}
Teaching Optics to students without a technical background lends itself particularly well to activities-based methods. Restricting the discussion to a lecture format, even if demonstrations are included, misses the opportunity to have students directly investigate something which is, quite literally, right before their eyes. At Southern Illinois University Edwardsville, we have redeveloped a course, entitled "Light \& Color," to be activities-based. Using hardware purchased specifically for this purpose, we have developed a set of student activities which are integrated into the syllabus. This course has been taught twice under the new paradigm with encouraging results. The activities developed are described and discussed and their impact on student performance is presented.
\end{abstract}

\section{Introduction}

With the creation of a Master's degree in Photonics at Southern Illinois University Edwardsville (SIUE), a significant number of courses in Optics and related fields at advanced levels were created and faculty specializing in research in various branches of Optics were hired. In order to make the activities of these faculty of more direct benefit to SIUE's non-technical students, the program in Photonics is complemented by a lower-division course entitled "Light \& Color." The purpose of the course is to teach elementary optics to students with little or no background in math or science.

The topics covered in the course include: Basic wave phenomena, the nature of light as an electromagnetic wave and methods of its creation, the eye and the relation of light to visual perception, color theory (additive and subtractive), the ray approximation, laws of reflection and refraction, basic optical devices, polarization of light, and optical phenomena in nature. The overall goal of the course is to demystify everyday experiences and to increase the students' understanding of uses of light as a tool.

This course fulfills group requirements for students in a variety of non-technical majors. The overwhelming majority of the students in the course are drawn from three majors: Art \& Design, Elementary Education, and Liberal Studies (a generic major in which the students develop an interdisciplinary program of study). As such, the level of preparation in mathematics of students in the class is extremely low. It has been found that explanations including even rudimentary mathematics will not be understood by a majority of the students in the class. Further, phenomena which are described, even if the description includes illustrations, are poorly absorbed. Classroom demonstrations have been observed to mitigate these problems only slightly.

These problems are common throughout Physics education, particularly when the students lack technical preparation. In Optics, however, many of the phenomena are directly visible under proper circumstances which are easily realized. To take advantage of this, we have begun redeveloping the curriculum of this course to be activitiesbased. Students explore the concepts at the core of the course via hands-on activities undertaken in small groups (3-4 students per group).

After the authors received an internal grant from SIUE (Excellence in Undergraduate Education Grant Number 0612) to acquire hardware necessary for this modification to the curriculum, approximately $1 / 6$ of the class meetings in the Fall, 2005 semester were devoted to activities. In the Fall, 2006 semester this fraction was increased to more than $1 / 3$ of class meetings. It was found that the level of comprehension of the subject was increased over that in prior semesters. Further, we were pleasantly surprised to note that the amount of material covered, overall, was utterly unaffected by replacing this fraction of lectures with activities.

Tenth International Topical Meeting on Education and Training in Optics and Photonics, edited by Marc Nantel, Proc. of SPIE Vol. 9665, 96651I · C 2007 SPIE, OSA, IEEE, ICO doi: $10.1117 / 12.2207325$ 


\section{Project overview}

The development of the new curriculum was undertaken by the authors. One of the authors (Glassman) holds a Ph.D. in Optical Sciences and was the Professor of record for the course. He set the overall goals for the course and defined and prioritized the key concepts it was desired that the students master via the course. The other author (Lindell) collaborated on the development of the activities and oversaw the collection of data for assessment.

The following key concepts were identified as candidates for benefit from the addition of an active learning component:

- Basic electrostatic interaction—charge and Coulomb’s law

- Oscillatory behavior—period, frequency, and amplitude of oscillations

- Wave motion —-wavelength, period, frequency, amplitude, and propagation speed of traveling disturbances

- Differences between spectral properties of light sources

- Human color perception— - "primary colors” as a manifestation of human trichromatism

- $\quad$ Additive color mixing

- $\quad$ Subtractive color mixing

- Light in the ray approximation-laws of reflection and refraction

- Basic optical devices—prisms, lenses, and mirrors

- Practical optical devices—-telescopes and microscopes

For each of the above concepts, we sought to enhance the students' learning experience by creating activities which would guide students to a more intuitive grasp of the concepts. The "Interaction Sessions" incorporating these activities replaced approximately one lecture per week for approximately half of the semester in the Fall, 2005 semester (the class met three times per week) and one lecture per week for almost the entire semester in the Fall of 2006 (in which the class met twice per week). Some activities spanned more than one class period. Conventional lectures, augmented by extensive demonstrations performed by the professor, were used in the remainder of the class meetings. It is worth reiterating that, despite the fact that the total number of traditional lectures in the course was reduced by approximately $20 \%$ in 2005 and more than $30 \%$ in 2006, the total amount of material covered in the semester was identical to that covered in previous, lecture-only semesters taught by the same professor.

\section{Course structure}

The overarching goal of the course is to increase the understanding that students have of the world they perceive visually. One crucial meta-concept that we wrestle with teaching students is that vision is a mechanical act. A specific study of student preconceptions about the nature of vision remains for future work. However, anecdotal observation and discussion with students leads us to infer that the students (and, one can presume, the bulk of the population as a whole) regard vision as an avenue of direct perception. This is exacerbated by the false concept frequently taught at the primary school level that "all colors are made from a combination of primary colors," relating the role of primaries to the light rather than to the physiology of the human eye. We worked to lead the students to an understanding of color perception as an interaction between light of various intensities which depend on wavelength and a set of light-sensitive cells in the eye that interact with the light with a set of sensitivities which are also wavelength dependent. But this sort of understanding is contingent upon understanding basic properties of light itself.

In order for students to gain insight into the behavior of light, it is essential that they obtain some understanding of its basic nature as an electromagnetic wave. While an explanation of this phenomenon in the context of Maxwell's equations is significantly beyond the level of this course, some understanding can be motivated by beginning with 
an exploration of classical electrostatics. While far from giving a complete picture of classical radiation, this at least motivates the notion of force at a distance. To make that force oscillatory is only a modest step. (Ampere's law and Faraday's law are presented via demonstrations to add the necessary magnetic force to the picture.)

We noted that students have tremendous difficulty mastering the concepts of frequency, period, and amplitude essential to any reasonable discussion of waves. Before attempting an exploration of wave propagation, these concepts need to be given concrete meaning for the students or subsequent discussion lacks context. So the class's exploration of electrostatics is followed by one of oscillatory motion.

The propagation of waves in the context of single pulses is next explored. This gives students a chance to develop their understanding of time-retarded effects and the concept of propagation speed for a disturbance as distinct from the speed of bulk motion of an object. Only after oscillations and wave propagation are explored separately is the notion of a traveling wave, driven by an oscillatory force, introduced.

Only after electromagnetic forces, oscillatory motion, and wave propagation are fully digested is electromagnetic radiation introduced. Spectra of atomic discharges, fluorescent sources, and blackbodies are studied by the students directly. A tool that was frequently employed, throughout the course, to aid the students in understanding the spectral composition of light was a small diffraction grating. Each student was given one of these at the beginning of the semester with instruction to bring it to every class session. The students were permitted to keep the gratings at the end of the semester. (Students were told that they would have to pay $\$ 1.00$ for a replacement grating if they lost the one they were given. None were lost in two semesters.) The professor, therefore, could at any time call upon the entire class to take out their gratings to observe something of interest. Students were strongly encouraged to make casual observations using their gratings outside of class as well.

Having established the nature of light as a traveling wave of oscillating electromagnetic force the role of the human eye as a mechanism of perception is introduced. Frequent recourse to the above-mentioned diffraction gratings is made. The limited range of wavelengths available to human perceptions is explored using a blackbody source (a filament lightbulb with a dimmer). Trichromatism is then explored. We have found that leading the students through the "unlearning" of preconceptions and misconceptions about vision makes this phase of the course the most challenging. The opportunity to have the students see for themselves that a "white" source composed of primary colors and an approximately white blackbody appear the same while having profoundly different spectral properties aids them in this process tremendously.

With trichromatism at least accepted, if not fully integrated, both additive and subtractive color mixing can be explored by making regular appeal to stimulation of cone receptors as the true definers of "color." Students examine various light sources through combinations of filters both directly and after dispersal by their diffraction gratings. Since the students were presented with the relationship between subjective response and objective source from the outset of their encounters with the concept of color, mixing can be explored in that context very naturally. This also allows for a brief discussion of photographic storage media.

The ray model of light is introduced very late in the course compared to the approach traditionally used in courses such as this. We noted that many authors begin with light in the ray approximation only to explore its underpinnings as a wave phenomenon later. We have found that approaching from the "bottom up" is less jarring to students. They discover the ray approximation, and its ability to lead to the concept of an image, through an exploration with pinhole viewers. Since they discovered the phenomenon of wave propagation, the speed of that propagation, and the relationship of that propagation to wave frequency and wavelength earlier in the course, the introduction of reflection and refraction flows naturally from the exploration which led to the ray model's introduction. Further, since their view of wave propagation was motivated by a basic exploration of electrostatic forces, the introduction of the effects of ponderable media requires only a modification of what they have previously learned and not the acceptance of a statement, made without context, about the effect of such media on the speed of light and its direction of propagation.

Explorations of basic optical components and their role in practical devices follow directly from the examination of general properties of light in the ray approximation. When exploring imaging devices, particular emphasis is placed on distinguishing between images formed directly in the viewer's eye and those formed on an external surface. We have found that students are particularly pleased to understand common eye diseases (e.g., myopia and hyperopia) 
and the role of corrective lenses. They seem to enjoy very much the demystification when we decipher an ophthalmologist's prescription!

A brief exploration of polarization is made particularly easy by the plethora of common devices which utilize polarization to achieve their goals. In both semesters, students have independently conceived of the notion of polarization when the original discussion of the creation of electromagnetic waves took place. Since they are presented with the idea of light as being a traveling disturbance resulting from oscillating charges at the outset, they very naturally consider that the direction of the oscillation must matter in some way. So the discussion of polarization effects and an exploration by the students of those effects is almost an afterthought.

The course concludes with an exploration of blue skies, rainbows, and other atmospheric phenomena. Again, the demystification of these is something that many seem to find very satisfying.

\section{Specific activities}

We will outline the activities introduced into the course so far. Others are under development and will be added to the course when it is next taught (by Glassman) in the Fall, 2007 semester. To present them in full detail is beyond the scope of this paper. We must hasten to note that some of these activities were developed by other researchers and have been taken from the literature while others were developed by us or our colleagues for use in other courses. The work presented here is the development of the curriculum around the stated activities rather than the development of the activities themselves.

As of this writing, the activities used are:

1. "Exploring Force at a Distance"- students perform the classic "sticky tape" activity to observe Coulomb's law and to motivate the concept of "charge."

2. "Exploring Oscillatory Motion”- students build pendula and make observations of their frequencies, periods, and amplitudes.

3. "Pulse Propagation" - students use long springs to observe pulse propagation rates. By creating pairs of pulses, they also observe destructive and constructive interference.

4. "Exploring Intensity Distribution”- using their diffraction gratings, the students observe a variableintensity filament lamp. They extract perceived intensity distributions for the lamp at different brightnesses. Doing this, they learn about blackbody spectra and begin to recognize that their own eyes react differently to different wavelengths. They also observe atomic discharge sources and a fluorescent source.

5. "Understanding Color Mixing—Part I”—using “Color Makers” (Fisher Scientific part \#S65092), boxes with red, green, blue, and white LEDs controllable with potentiometers, students discover that subjective perception of color can be quite different from spectral reality. They observe the Color Makers through their diffraction gratings and see the limited spectra of the output. By changing their distances from the Color Makers, they are able to see the source seem to transition from tri-colored to white. This introduces them to the concept of trichromatism.

6. "Exploring Filters-Part I" - students use colored filters to explore additive color mixing. This is put in the context of trichromatism as part of the exercise.

7. “Exploring Filters—Part II"-students again use colored filters, this time to explore subtractive color mixing. The addition of the diffraction gratings to the process particularly aids their understanding in the context of trichromatism.

8. "Exploring Rays—Part I"—students use "Ray Boxes” (Fisher Scientific part \#S42580B) to explore the behavior of rays at surfaces. Laws of reflection and refraction are inferred. Dispersion is observed. Total internal reflection is observed, although discussion of it is deferred until a future class. 
9. “Exploring Rays_-Part II: Imaging”-pinhole viewers (Pasco part \#OS-9498A) are used to motivate the concept of an image as a mapping of rays from an object point to an image point. The ability to create an approximate image with an approximate mapping is explored. (Students frequently expect things to be allor-nothing and so benefit from being shown that approximate results can be acceptable.)

10. "Exploring Rays_-Part III: Imaging with Lenses"—-students create images with lenses on a simple optical bench. They observe real images and begin to understand raytracing. Concepts such as magnification and inversion are explored. Also, virtual images are explored. Combinations of lenses are used as well as single lenses.

11. "Exploring Telescopes"- students build simple refracting telescopes. They explore the use of these without the eyepiece at first, forming a real image on a sheet of paper and then on their own cornea. We have noted that many students (including those at advanced levels) fail to consider that a view-through device is intended to form a real image on a human retina and so must consider the presence of the lens/cornea of the viewer's eye. In this activity, the students learn the utility of zero-power imaging devices.

It should be noted that some of the above activities require less than a full class period while others span more than one. Other activities are under development and are expected to be implemented in the Fall, 2007 iteration of the course.

In addition to the direct advantages of active learning, we have found that the addition of activities to the curriculum tremendously enhances student engagement. In particular when the course is taught in the evening (as it was in 2006), the need to enliven a group of students who arrive already exhausted is profound. It has been noticed that if even a portion of a class period is devoted to an activity the level of student engagement and apparent comprehension is greatly increased for the remainder of the session.

\section{Evaluation of Student Learning}

To assess the effectiveness of these techniques, we performed a Primary Trait Analysis [1] (PTA) on each of the course exams. This analysis involves totaling students scores for each of the different subjects covered during the course. In addition we tallied scores for performance on the integrated portions on each of the three exams. All attempts were made to make all sections uniform, however this was not always possible. We judged the instruction to be successful if the PTA were above $70 \%$, moderately successful if above $60 \%$ and not successful if below $60 \%$. While we are disappointed with the results for Wave Motion, it is worth noting that this level is markedly higher than that for the same topic when taught without activities in the previous years. Before the introduction of activities, the level of understanding of students was essentially zero. Nevertheless, we hope for improvement in this area in the future. (Mastery of "Practical Optical Devices" was not explicitly assessed.)

\begin{tabular}{|c|c|c|}
\hline Topic & $\begin{array}{l}\text { Avg. Percent Total (STDEV) } \\
\text { Fall 2005; N=33 }\end{array}$ & $\begin{array}{l}\text { Avg. Percent Total (STDEV) } \\
\text { Fall 2006; N=26 }\end{array}$ \\
\hline $\begin{array}{l}\text { Basic electrostatic interaction-charge and } \\
\text { Coulomb's law }\end{array}$ & $57.42 \%(25.19 \%)$ & $68.91(26.19 \%)$ \\
\hline $\begin{array}{l}\text { Oscillatory behavior-period, frequency, } \\
\text { and amplitude of oscillations }\end{array}$ & $66.46 \%(22.25 \%)$ & $93.96 \%(22.20 \%)$ \\
\hline $\begin{array}{l}\text { Wave motion-wavelength, period, } \\
\text { frequency, amplitude, and propagation } \\
\text { speed of traveling disturbances }\end{array}$ & $59.64 \%(44.15 \%)$ & $44.78 \%(33.01 \%)$ \\
\hline $\begin{array}{l}\text { Differences between spectral properties of } \\
\text { light sources }\end{array}$ & Not Evaluated & $83.74 \%(29.48 \%)$ \\
\hline $\begin{array}{l}\text { Human color perception-“primary } \\
\text { colors” as a manifestation of human } \\
\text { trichromatism }\end{array}$ & Not Evaluated & $70.79 \%(22.39 \%)$ \\
\hline Additive color mixing & 70.91\% (16.54\%) & 72.94\% (28.11\%) \\
\hline Subtractive color mixing & $33.94 \%(35.06 \%)$ & $60.22 \%(34.82 \%)$ \\
\hline $\begin{array}{l}\text { Light in the ray approximation-laws of } \\
\text { reflection and refraction }\end{array}$ & $69.76 \%(28.14 \%)$ & $68.02 \%(24.44 \%)$ \\
\hline $\begin{array}{l}\text { Basic optical devices_-prisms, lenses, and } \\
\text { mirrors }\end{array}$ & Not Evaluated & $68.70 \%(30.42 \%)$ \\
\hline
\end{tabular}




\section{Conclusions and plans for future development}

Our intention is to increase the number of activities when this course is next taught (Fall, 2007). We hope to have at least one active-learning session per week in this next iteration. Also, the activities that have been used previously will certainly be revisited and revised to improve their effectiveness. Teaching in an active-learning environment makes particular demands on the instructor, distinct from those of a traditional lecture course. We expect improvement in student mastery of the subject as the professor's mastery of this distinct skill set increases.

We are very pleased with the successes of the revised curriculum. The measurable aspects of these improvements, described above, tell only part of the story. An increased level of student satisfaction is obvious to the instructor and to colleagues who have observed the class. The fact that the improvement in student performance came at no cost to course breadth is a particular source of pride: We taught them just as much, we just taught them better!

\section{Acknowledgements}

Support for this project was provided by SIUE Excellence in Undergraduate Education Grant Number 06-12. Assistance was given to both authors by several undergraduate students, most notably Nathan Motl, Jeremy Wood, Jacob Carlson, and Curt Bradley, whom we thank for their aid.

\section{Reference}

[1] B Walvood and L. P. McCarthy, 1990. Thinking and writing in college. Urbana, IL: National Council of Teachers of English 